\title{
Premiers diagnostics d'un bassin versant pour une annonce de crue
}

\author{
J.M. Masson \\ Responsable de la filière Eau \\ de l'Institut des sciences de l'ingénieur \\ Laboratoire d'hydrologie mathématique, Montpellier
}

\begin{abstract}
Le problème du premier diagnostic d'un bassin versant fait l'objet du chapitre 2 du Guide de prévision des crues. Quelques mois après la sortie de cet ouvrage, il n'est guère possible d'apporter des idées nouvelles. L'essentiel de cette communication s'inspire donc du guide, en insistant sur l'aspect hydrologique des prévisions, aspect que le développement des méthodes et des techniques pourrait parfois faire oublier.
\end{abstract}

\section{Facteurs des crues sur les bassins ver- sants}

Un abaque déjà ancien (Sogreah, 1968), proposé pour estimer les risques de crue sur les petits bassins versants, nous renseigne sur les facteurs qui agissent sur l'importance des crues.

\subsection{Les possibilités de pluies intenses}

Les pluies intenses sont la cause directe de crues importantes. Il faut avoir vécu des pluies supérieures à $40 \mathrm{~mm} /$ heure pendant 5 ou 6 heures pour savoir que l'eau peut monter au-dessus de la surface de terrains "plutôt perméables ", cette lame d'eau arrivant à se maintenir dans des conditions topographiques difficiles (sommet des buttes).

De tels événements pluvieux ne sont pas exceptionnels sur le pourtour de la Méditerranée. On peut y observer le phénomène suivant : sur des sols nus réputés perméables, l'énergie cinétique et l'effet de "splash " des pluies intenses forment une organisation pelliculaire de surface quasiment imperméable dite croûte de battance. Ce phénomène est fréquent en région sahélienne.

Dans le Guide de prévision des crues, la hauteur de pluie journalière décennale a été choisie comme indicateur des possibilités de pluies intenses. Une hauteur inférieure à 60 millimètres correspond à des régions (Nord et Ouest de la France) où des averses supérieures à $100 \mathrm{~mm}$ en quelques heures sont exceptionnelles. Si elles se produisent, ce sera pendant la saison chaude (pluies convectives avec orages) et sur des étendues relativement limitées. Ainsi sur l'Ornain (affluent de la Saulx tributaire de la Marne) au mois d'août 1972.

\subsection{Les caractéristiques des bassins versants}

Les caractéristiques des bassins versants qui influencent les crues sont nombreuses mais leurs effets sont difficiles à quantifier.

Seul descripteur vraiment quantitatif d'un bassin versant, sa superficie est un facteur multiplicatif. Son estimation ne pose problème qu'en présence d'un réseau karstique ou pour de très petits bassins (Dupraz, 1984).

Les pentes des versants et des cours d'eau influencent la rapidité de concentration de l'écoulement. Les indicateurs globaux qui les apprécient ne sont que des moyennes un peu spéciales recouvrant des situations locales diverses et n'ont donc pas la même signification hydraulique que la pente d'un canal par exemple.

The first diagnostics of a drainage area relating to a flood warning After underling the importance and rapidity of floods depending on possible occurrences of heavy rainfall and the characteristics of the drainage area, the specific nature of each flood is thoroughly examined. The inventory, acquisition and analysis of the available data, as well as a site inspection, are strictly necessary so as to specify the required and possible periods of time, as well as the time intervals to be taken into consideration. An ordinary hydrological method is proposed for forecasting the flow rates from rain. This method uses only two parameters from which it is possible to assess the variation direction depending on the scale and nature of the floods. 
En ce qui concerne la nature du sol et du sous-sol, la classification simple proposée dans le Guide de prévision des crues est réaliste. marnes et argiles donnent des sols plutôt imperméables qui favorisent le ruissellement, tandis que la présence d'un aquifère et son importance distinguent les sous-sols imperméables et perméables. Un sous-sol imperméable ne signifie par forcément pour un bassin versant une capacité d'absorption très faible. Ainsi un socle métamorphique peut être surmonté d'une couche plus ou moins épaisse de produits d'altération qui stockent beaucoup d'eau avant d'être saturés. A la suite des crues des 25-30 septembre 1907, la rétention des bassins cévenols pouvait être estimée à près de $200 \mathrm{mil}$ limètres sur les 400 tombés (Pardé, 1933); mais 8 jours plus tard, l'Ardèche écoulait plus de $80 \%$ d'une nouvelle averse importante. Sur l'ouest du Massif Central, la capacité d'absorption des bassins serait de l'ordre de $100 \mathrm{~mm}$.

\subsection{Rapidité des crues}

Les possibilités de prévision dépendent de la rapidité des crues. Avec des précipitations intenses, la crue est toujours rapide. Les exemples de crues rapides ne manquent pas. Citons l'Allier à Vieille-Brioude $\left(2260 \mathrm{~km}^{2}\right)$ en septembre 1866 avec une montée de près de 9 mètres en une dizaine d'heures, un record homologué appartenant au Vidourle à Quissac $\left(217 \mathrm{~km}^{2}\right)$ où en octobre 1958 on a observé 4,5 mètres en 45 minutes, soit $10 \mathrm{~cm} / \mathrm{min}$ (Jacquet, 1959).

Des crues lentes formées d'eau initialement infiltrée suintant dans les fonds de vallon résulteront de pluies faibles mais prolongées, tombant sur un bassin versant au sol et sous-sol plutôt perméable, avec un relief faible (Saône, Seine, Marne).

\subsection{Quelques ordres de grandeur}

Pour fixer les idées, rappelons les valeurs de débits pouvant être atteints en France selon la situation et la surface des bassins. Ces valeurs (Pardé, 1933), établies depuis plusieurs décennies, me semblent toujours d'actualité.

Débit spécifique en $m 3 / \mathrm{s} / \mathrm{km}$ ?

\begin{tabular}{|l|c|c|c|c|}
\hline \multicolumn{1}{|c|}{ Surface } & 100 & 500 & 3000 & 10000 \\
\hline Réglion méditerranéenne & $10-25$ & $5-15$ & $2-5$ & $1-2.5$ \\
\hline Montagnes & $1.5-2.0$ & $0.8-1.5$ & $0.6-1.90 .35-0.8$ \\
\hline Pla1nes & $0.3-0.5$ & $0.15-0.3$ & $0.1-0.25$ & $0.05-0.15$ \\
\hline
\end{tabular}

Comme il est difficile d'être précis en matière de fréquences rares, disons qu'il s'agit de valeurs autour d'une période de retour ponctuelle de 100 ans, mais qu'on peut observer beaucoup plus fréquemment à l'échelle d'une région. Nous savons par exemple qu'une pluie journalière, décennale ponctuellement, se produit en moyenne une fois tous les 8 mois dans la région pari- sienne. Or les bassins versants de quelques dizaines de $\mathrm{km}^{2}$ sont des milliers en France où on observe chaque année des dizaines d'orages accompagnés de pluies importantes. Malgré la non coïncidence, heureusement fréquente, de l'emprise de l'orage et des bassins versants, des crues importantes pour ces superficies s'y produisent fréquemment, peut être plus d'un débit de pointe de période de retour 20-50 ans en moyenne par an, souvent en dehors des points de mesure il est vrai. Ces expériences subies sont-elles bien utilisées pour augmenter notre base de connaissances?

\subsection{Personnalité de crues}

Dès qu'il est un peu grand, un bassin versant est rarement homogène vis-à-vis des facteurs qui influencent les crues; suivant que l'épicentre de l'averse se trouve sur telle ou telle partie du bassin, que les périodes de forte intensité se trouvent au début ou à la fin de l'averse, les crues seront plus ou moins importantes et iront plus ou moins vite. Il faut revoir à ce sujet les explications géométriques qui sont résumées sur une page d'un manuel d'Hydrologie bien connu (Réménieras, 1972). Dans ces conditions, une schématisation globale et simple du phénomène, telle qu'on peut la concevoir au stade d'un premier diagnostic, sera forcément imprécise.

\section{Les observations}

\subsection{Inventaire et recueil}

Un diagnostic sérieux ne pouvant se concevoir sans utiliser les données des observations, il faut procéder très rapidement à l'inventaire des stations de mesure existantes ou ayant existé : mesures de hauteurs d'eau, de débits, des précipitations. En ce qui concerne les précipitations, on retiendra les postes jusqu'à $10 \mathrm{~km}$ au moins à l'extérieur des limites du bassin. Toutes les observations particulières (lignes d'eau, cote des délaissés,...) recueillies à l'occasion des grandes crues seront également inventoriées.

Une première décision peut intervenir à ce stade : en l'absence de mesures de débits, il est urgent de programmer des jaugeages. En matière de prévision des crues, la connaissance des débits est en effet une priorité rappelée en plusieurs endroits dans le Guide.

Il est judicieux de construire un graphique permettant d'appréhender d'un seul coup d'œil les périodes de fonctionnement des diverses stations et de s'assurer, dès le départ, que pendant la période couverte par les observations il n'y a pas eu de modifications importantes, ni dans les conditions de mesure, ni dans les conditions d'écoulement, en répondant à une série de questions dont on trouvera la liste (non exhaustive) dans le guide. Les répercussions de la non permanence sont souvent mal perçues. Ainsi une crue qui a donné un débit de pointe de $1000 \mathrm{~m}^{3} / \mathrm{s}$ sur l'Hérault à Agde dans les conditions anciennes, pourra donner un débit de pointe de $1500 \mathrm{~m}^{3} / \mathrm{s}$ si on endigue l'Hérault. Les fréquences attribuées aux débits à partir des observations antérieures ne sont plus valables. 


\subsection{Détermination des seuils et sélection des événements}

Le rapprochement des observations et des dommages constatés permet de fixer des seuils de hauteur ou de débit séparant les 3 états suivants : absence de nuisances - nuisances acceptables - dommages graves.

Ces seuils de hauteur ou de débit permettent une première sélection des observations pour les crues ayant atteint ou dépassé ces valeurs. Une analyse rapide de ces crues donne la valeur minimale des entrées du système (pluie ou débit amont) ayant conduit à un dépassement du seuil.

Une seconde sélection retiendra alors les observations correspondant aux entrées ayant dépassé ou atteint une valeur proche de ce minimum. Ne perdons pas de vue en effet que la prévision utilise les entrées du système (pluie, débit) pour anticiper ce qui va se passer à la sortie. Pour être fiable, elle doit donc déterminer les raisons qui font qu'une même entrée conduit ou ne conduit pas à une crue dommageable.

Les observations correspondant aux événements sélectionnés sont mises en forme et à cette étape se pose le problème de l'intervalle de temps à retenir. Cet intervalle de temps devra être contenu au moins 2 fois dans les temps de montée les plus courts des crues dommageables. Si cet intervalle est inférieur à 24 heures et que la pluie n'est mesurée qu'avec des pluviomètres, l'installation de pluviographes s'imposera et on devra attendre qu'ils aient enregistré quelques événements avant de pouvoir faire des prévisions.

Souvent à partir de quelques dizaines d'années d'observation, il ne reste que quelques dizaines d'événements qui peuvent être critiqués et analysés en vue du diagnostic.

\subsection{Critique des données}

Cette critique a déjà débuté avec la recherche des modifications dans les conditions de mesure et d'écoulements et avec l'examen des courbes de tarage.

Les méthodes élaborées utilisées habituellement pour critiquer les séries pluviométriques : double cumul, ellipses de contrôle des écarts cumulées, A.C.P. éventuellement, ne sont pas adaptées à la critique d'événements isolés. Elles ont été conçues pour détecter des différences systématiques sur les totaux annuels, différences dues généralement à des changements dans l'environnement de l'appareil (déplacement, constructions, arbres) qui affectent surtout les pluies peu intenses par l'intermédiaire du vent. Quel est l'intérêt pour l'annonce de crue de déterminer qu'à partir de telle année, tel poste donne un total annuel supérieur de $5 \%$ en moyenne à ce qu'il donnait avant parce qu'il a été déplacé d'un site venté à un site abrité ? Cette conclusion ne concerne pas les fortes pluies génératrices de crues et sur lesquelles en général le vent a peu d'influence.

On devra cependant vérifier la cohérence des observations au moyen de graphiques et cartes appropriées. On pourra par exemple constater que l'évolution des hauteurs observées sur un affluent est toujours en parfaite concordance avec les indications de l'échelle la plus proche de la rivière principale parce qu'elle est influen- cée par le remous de cette rivière. De même la cartographie des isohyètes d'une averse, pour les différents intervalles de temps retenus, permet de repérer d'éventuelles anomalies et conduit à consulter les documents originaux concernant les mesures.

Une visite sur le terrain est indispensable. Elle permet de se faire une idée de la représentativité des stations d'observation et de repérer des cas particuliers dont il est difficile de faire l'inventaire.

Ainsi, avant d'entreprendre l'étude de la prévision des crues sur la Loire à Feurs, une visite sur le terrain aurait permis de constater que l'échelle d'annonce de crue de Feurs est située à l'amont d'un barrage mobile; une discussion avec les gestionnaires aurait appris que ce barrage est abattu à l'estime pour des débits allant de $350 \mathrm{~m}^{3} / \mathrm{s}$ à plus de $700 \mathrm{~m}^{3} / \mathrm{s}$. Dans ces conditions, les observations anciennes des hauteurs d'eau ne permettent pas d'estimer les débits dans cette gamme de valeurs. Certes, pour les forts débits, le barrage est abattu et la relation hauteur-débit est bonne. Cependant, pour pouvoir estimer l'hydrogramme futur d'une crue qui débute, hydrogramme qui détermine le débit d'écrêtement du barrage de Villerest, il est indispensable de connaître le début de la crue avec un minimum de précision. Les observations anciennes ne permettant pas cette précision, la qualité des prévisions s'en ressent.

\section{Estimation des délais nécessaires et possibles}

Le chronogramme figurant dans le Guide de prévision des crues, indique clairement les 3 durées: durée de recueil des données - durée des opérations de prévision - durée des actions préventives, dont l'addition constitue le délai minimum avec lequel on doit anticiper la crue et qu'on appelle délai de sauvegarde.

Il faut comparer ce délai de sauvegarde au temps que la rivière laisse au prévisionniste et qu'on appelle délai de prévision. On estime ce délai de prévision par le temps de réaction du bassin qui est la durée séparant la fin du corps intense de l'averse de la pointe de crue, pour des averses dont le corps intense est sensiblement inférieur au temps de réponse du bassin. Le temps de réaction, imposé par la nature, est peu compressible. Avec des pluies exceptionnellement fortes, sa valeur diminue dans les rivières encaissées (Haut Allier, Haute Loire, Vézère), mais elle augmente en présence d'un champ d'inondation important (basse vallée de l'Hérault, Huveaune).

Si le temps de réaction est plus court que le délai de sauvegarde, l'annonce de crue sera inefficace et on cherchera les moyens susceptibles de raccourcir le délai de sauvegarde.

Quand la prévision utilise les débits d'une station amont, le délai de prévision est le temps de propagation. En première approximation, sa valeur est donnée par le décalage entre les pointes de crue amont et aval. Outre la distinction qu'il faut faire entre crues débordantes et non débordantes, le décalage entre les pointes ne représente le temps de propagation que si les apports des bassins intermédiaires sont faibles. 


\section{Quelques méthodes simples utilisables pour la prévision}

Les méthodes simples sont utilisables surtout quand la prévision utilise l'information de stations hydrométriques amont.

Dans ce cas, le temps de propagation $\Delta t$ étant déterminé, on peut porter graphiquement les valeurs amont et aval décalées du temps de propagation et tracer la courbe de la relation moyenne dans le nuage de points ainsi obtenus.

En matière de liaisons pluies-débits, le premier diagnostic peut consister en un tri des conditions d'occurrence des crues. Pour une période donnée de l'année, il est possible de tracer la courbe enveloppe des débits atteints en fonction de la pluie et des conditions initiales d'humidité. Un exemple est donné dans le Guide. Mais nous pensons que des méthodes un peu plus hydrologiques que les régressions peuvent être utilisées avec peut-être moins de risques.

La méthode que nous proposons s'inspire de ce qui a été fait sur la Vézère (Fabret, 1968). Nous en donnons ci-dessous le principe, sachant que les applications peuvent conduire à de nombreuses variantes.

$\mathrm{Si} \Delta t$ est le temps de réaction, cet intervalle est aussi celui sur lequel on considère la pluie. Une pluie efficace $\mathrm{Pe}(t)$ de $1 \mathrm{~m}^{3} / \mathrm{s}$ entre $t-\Delta t$ et $t$ donne :

- un débit $a$ faible au temps $t$

- un débit $b$ très supérieur à $a$ au temps $t+\Delta t$

- les débits décroissent ensuite de façon exponentielle telle que

$Q(t+n \Delta t)=b e-\frac{(n-1) \Delta t}{k}$

Ceci constitue la fonction de transfert du modèle, qui permet la prévision par l'expression :

$$
\begin{aligned}
Q(t+\Delta t)=x[Q(t)-a & \operatorname{Pe}(t)] \\
& +b \operatorname{Pe}(t)+a \operatorname{Pe}(t+\Delta t)
\end{aligned}
$$

avec $x=(1-\alpha) \simeq e^{-\alpha}$, en posant $\alpha=\Delta t / k$.

La fonction de transfert n'a qu'un paramètre à caler par optimisation, puisque la valeur de $k$ peut se déduire des décrues observées et que, compte tenu de la conservation des volumes, on a la relation :

$$
b=(1-x)(1-a)
$$

Pour passer de la pluie brute $P(t)$ à la pluie efficace $\mathrm{Pe}(t)$, on retient une relation à un seul paramètre $c$ :

$$
\operatorname{Pe}(t)=f(c, P(t)) \text {. }
$$

Il y a donc 2 paramètres à estimer : $a$ ou $b$ et $c$.

\section{Calage}

En utilisant l'équation de prévision sur les événements crues du passé, on cherche, pour chaque crue $i$ le couple des valeurs des paramètres $\left(c_{i}, b_{i}\right)$ qui donne les meilleurs résultats au sens d'un critère d'écart, celui par exemple qui minimise la somme des valeurs absolues des écarts entre les débits prévus et les débits observés.

En prenant pour valeur de $b$ la moyenne des $b_{i}$, on cherche à nouveau la valeur optimale de $c_{i}$ pour chacune des crues. Cette valeur $c_{i}$ variant d'une crue à l'autre, on s'efforce de trouver une liaison entre les valeurs de $c_{i}$ et l'état d'humidité du bassin au début de la crue, état caractérisé par les débits de la rivière ou les antécédents de pluie par exemple.

\section{Prévision}

Une crue s'annonçant, $c$ est estimé à partir de l'état d'humidité du bassin. Cette valeur est utilisée pour les 2 premières prévisions. Dès qu'on a observé 2 réalisations, la simplicité de la formule de prévision permet, grâce à un contrôle arrière, d'estimer la valeur de $c$ qu'il aurait fallu prendre pour rendre correctes les 2 premières prévisions. Cette valeur est alors utilisée pour la prévision suivante. Par la suite, la valeur de $c$ est constamment réestimée à partir des deux dernières réalisations.

En cas de précipitations exceptionnelles et dans les vallées encaissées, on peut constater que $\Delta t$ diminue tandis que la valeur de $a$ augmente. Quand de tels événements ont été observés (en septembre 1980 sur la Haute-Loire), ils sont traités séparément.

La principale qualité de la méthode proposée, c'est d'avoir peu de paramètres dont on connaît le sens de variation.

\section{Conclusion}

Etant donné la complexité des problèmes associés aux crues, chaque bassin versant est un cas particulier. Dans ces conditions, la structure linéaire d'un exposé convient mal à l'établissement d'un diagnostic qui serait davantage du ressort d'un système expert.

L'Hydrologie est une science expérimentale historique. La prévision des crues est maintenant possible parce que, dans le passé, certains ont perçu la nécessité de constituer et maintenir un réseau d'observations.

L'information apportée par l'observation des grandes crues est irremplaçable. Pour quelques unes d'entre elles, la S.H.F. est à l'origine de documents de synthèse remarquables qui sont une mine de renseignements. Sauf à admettre que depuis une vingtaine d'années il n'y a plus de grandes crues, cette source documentaire semble tarie et nous ne pouvons que le regretter.
Adresse de l'auteur

Monsieur J.M. Masson

Laboratoire d'hydrologie mathématique

USTL

place Eugène Bataillon

34060 Montpellier Cedex

Tél. : (16) 67.63.33.39 


\section{Bibliographie}

DuPRAZ C., 1982. - Bilans des transferts d'eau et d'éléments minéraux dans trois bassins versants comparatifs à végétations contrastées (Mont Lozère, France).

Thèse de Docteur-Ingénieur, Université d'Orléans.

FABRET Cl., 1968. - Annonce des crues du bassin de la Vézère. Calcul des débits à partir des précipitations. Bilan hydro. logique.

Ecole Nationale des Ponts et Chaussées. Troisième session de recyclage en hydrologie.

JACQUET J., 1959. - Les crues d'automne 1958 sur le Vidourle. La Houille Blanche, $\mathrm{n}^{\circ}$ A-1959, p. 698-714.

Normand D. et Veyrat P., 1968. - Etude de synthèse des crues en France au débouché de bassins versants de 1 à $100 \mathrm{~km}^{2}$. Rapport Sogreah R 9861 pour le Service spécial des autoroutes. Agence du Sud-Est. Mâcon.

PARDÉ M. 1933. - Fleuves et rivières. Armand Colin. Collection U2. $5^{\mathrm{e}}$ édition, juillet $1968,241 \mathrm{p}$.

RÉMÉnIÉRAs G., 1972. - L'Hydrologie de l'ingénieur. Collection de la Direction des études et recherches d'EDF, Eyrolles, Paris. $3^{\mathrm{e}}$ édition, $456 \mathrm{p}$.

Débit spécifique en $\mathrm{m}^{3} / \mathrm{s} / \mathrm{km}^{2}$ 
Le Président: Je remercie M. Masson de son exposé. Nous allons passer aux questions. Je voudrais moi-même faire une remarque : M. MASSON nous a parlé notamment de la critique des données. Je voudrais vous rapporter ici une anecdote que j'ai vécue, que certaines personnes présentes dans la salle connaissent également, et qui est le cas contraire de ce que vous avez présenté. Non pas que ce que je vais dire soit contradictoire avec ce que vous avez dit, mais c'est un complément. Certains d'entre vous ont entendu parler de la crue de 1960 dans le bassin de la Corrèze; c'est une crue extrêmement importante et que, personnellement, j'ai beaucoup étudiée. En utilisant l'arsenal que vous avez présenté, Monsieur Masson, et qui fonctionne assez bien pour la plupart des crues, nous n'étions jamais arrivés à expliquer la crue de 1960. Il se trouve que la personne qui s'occupait des jaugeages à Périgueux à l'époque m'avait expliqué que tous les limnigraphes avaient été emportés, mais que lui, tel jour, à telle heure, avait constaté telle cote. Pendant six mois, je ne lai pas cru parce que je n'arrivais pas à expliquer cette cote par les méthodes que j'avais trouvées dans les livres existants, et c'est le jour où je l'ai cru que nous avons progressé.

Ceci montre qu'il faut, par des méthodes graphiques ou autres, telles que nous les a montrées M. Masson, critiquer de très près les données dont nous disposons. Mais parfois, avant de rejeter une donnée suspecte, il faut voir si, par hasard, elle ne serait pas exacte. J'en profite pour dire que, lorsque ces phénomènes sont traduits sous forme linéaire - M. MASSON nous l'a bien expliqué - cela marche très bien jusqu'à la pluie décennale, au maximum cinquantennale, et après les phénomènes s'accélèrent et je dirais que c'est un autre type de crue. Cela aussi, il faut l'avoir en tête. Le diagnostic du bassin est très difficile à faire dans ce cas-là puisqu'il s'agit de crues très rares pour lesquelles on n'a pas forcément les données. Quelqu'un souhaite-t-il poser une question à M. MASSON?

M. PARSY: Je pense que M. Masson aurait pu insister sur les questions d'hydrogéologie du bassin versant. Lorsqu'on est dans des systèmes de roches imperméables, mais fracturées, on peut avoir des différences d'hydrogrammes notamment entre ceux des petites crues et ceux des fortes crues : c'est le cas de la Corrèze au niveau de Tulle. Il peut y avoir aussi des systèmes karstiques dans lesquels des galeries ou des cavernes peuvent se siphoner dans une autre rivière et, dans ce cas, les hydrogrammes habituels relatifs à des crues fréquentes n'ont plus aucun rapport avec ceux que l'on mesure lors de crues graves.

M. MASSON : Je n'ai pas pu dire tout ce que j'aurais voulu dire étant donné le temps limité. Je peux citer aussi l'exemple d'une crue sur le Lez à Montpellier. La température de l'eau a montré que la pointe de crue a été causée par l'eau souterraine. Quand on parle d'écoulement rapide, il ne s'agit pas forcément de ruissellement. Il faut faire attention à cela.
M. DUBAND : Je ne suis pas convaincu par l'affirmation : « les petites crues et les grandes crues ont une typologie particulière ". Je crois qu'il y a deux phénomènes : il y a le phénomène de la fonction de production, c'est-à-dire le rendement d'une pluie, et le phénomène de son étalement d'amont en aval. Quand on parle de crue de moyenne importance et d'une autre famille d'hydrogramme de crue, est-ce vraiment fondé ?

M. P.A. ROCHE : Il y a une différence pratique évidente : pour comprendre le comportement des petites crues, on dispose de données assez nombreuses, ce qui n'est pas le cas pour les grandes crues. Le comportement hydrologique dans ces situations extrêmes n'est peut-être pas fondamentalement différent de celui des situations plus communes, mais les paramètres en sont plus incertains. La tendance naturelle est alors d'extrapoler ce qui est bien connu, mais cette extrapolation ignore l'évidence que le système mobilisé par l'écoulement dans le cas des fortes crues est plus étendu, et plus rempli, sans doute plus linéaire que celui qui est concerné par les petites crues.

Le Président: Je voudrais essayer de donner des éléments de réponse. Il est bien évident que le système est continu : on ne passe pas d'un système $\mathrm{A}$ à un système $\mathrm{B}$ d'un seul coup.

D'autre part, M. MAsson l'a bien rappelé, il y a des cas où les temps de propagation s'accélèrent et des cas où les vitesses de propagation diminuent. Pourquoi ? Parce que, en gros, la vitesse de propagation de l'onde de crue est proportionnelle à la pente de la courbe de tarage et inversement proportionnelle à la largeur du plan d'eau en surface. Selon que c'est un paramètre ou l'autre qui l'emporte, la crue s'accélère au fur et à mesure qu'elle est plus importante ou bien, au contraire, elle ralentit.

Si l'on se place dans des bassins versants relativement petits - j'ai cité la Corrèze à Brive, je peux citer la Loire en amont de Brive-Charensac; cela fait à peu près 1000 à $2000 \mathrm{~km}^{2}-$ jusqu'à la crue décennale on ne fait pas trop d'erreur en prenant un modèle linéaire mais, ensuite, les phénomènes s'accélèrent. Par exemple, à Brive-Charensac sur la Loire, on a en moyenne un $\Delta t$ de 4 heures, c'est-à-dire qu'on dispose d'un délai de préavis de $4 \mathrm{~h}$ (dans la pratique, c'est un peu moins : il faut envoyer le message). Quand s'est produite la crue de 1980 sur la Loire, le $\Delta t$ mesuré était au maximum de $2 \mathrm{~h}$. Parce que tout s'accélère. La méthode linéaire de prévision fait faire une erreur de $50 \%$. Le phénomène est évidemment continu. Je me plaçais dans l'hypothèse de méthodes grossières d'appréhension du phénomène. Si l'on a une méthode de prévision qui ressemble à ce qui vient d'être présenté par M. MASSON, cela marche dans beaucoup de cas, mais, lorsqu'un phénomène un peu exceptionnel arrive, il faut penser qu'une adaptation significative est nécessaire. Voilà ce que je voulais dire, quand je parlais de deux catégories de crues pour un même bassin versant. 